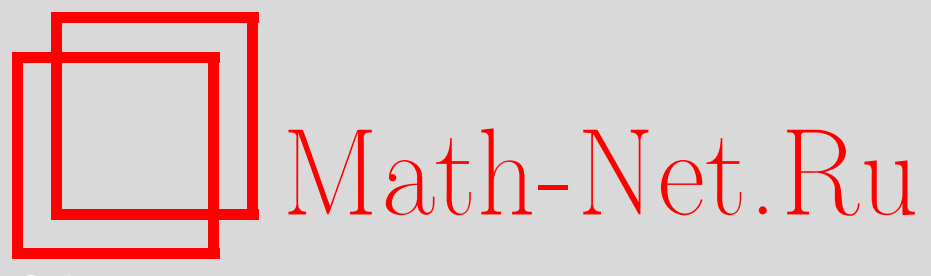

А. М. Каменецкий, Рациональность производящих функций цикловых ладейных полиномов и цикловых перманентов тёплицевых матриц и циркулянтов, УМH, 2007, том 62, выпуск 6, 175-176

DOI: https://doi.org/10.4213/rm8551

Использование Общероссийского математического портала Math-Net.Ru подразумевает, что вы прочитали и согласны с пользовательским соглашением http://www . mathnet.ru/rus/agreement

Параметры загрузки:

IP: 54.89 .56 .158

26 апреля 2023 г., 09:58:12

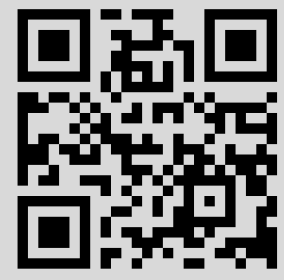




\section{Рациональность производящих функций цикловых ладейных полиномов и цикловых перманентов тёплицевых матриц и циркулянтов}

\section{А. М. Каменецкий}

Пусть $T_{n}^{(k)}$ - тёплицева $(n \times n)$-матрица, имеющая единицы на диагонали $j-i=k$ (где $i$ - номер строки и $j$ - номер столбца) и нули в остальных местах, и пусть $P_{n}=T_{n}^{(1)}+T_{n}^{(1-n)}$ - циркулянт. Зафиксируем некоторые целые $r$ и $t, 0 \leqslant r \leqslant t$, и рассмотрим матрицы $A_{n}=A_{n, t}^{\langle r\rangle}=\sum_{i=-r}^{-r+t} a_{i} P_{n}^{i}$ и $B_{n}=B_{n, t}^{\langle r\rangle}=\sum_{i=-r}^{-r+t} a_{i} T_{n}^{(i)}$, где $a_{i}$ - элементы некоторого коммутативного кольца (их можно считать формальными коммутирующими переменными). В настоящей статье вычисляются цикловые ладейные полиномы $R\left(x, z ; A_{n}\right)$ и $R\left(x, z ; B_{n}\right)$, а также цикловые перманенты $\operatorname{per}\left(z ; A_{n}\right)$ и $\operatorname{per}\left(z ; B_{n}\right)$ матриц $A_{n}$ и $B_{n}$. Вычисление этих многочленов решает задачу перечисления перестановок с ограниченными позициями и фиксированным числом циклов [1].

Tеорема 1. Существуют не зависящие от $n$ квадратные матрицы $K_{t}^{\langle s, r\rangle}=$ $K_{t}^{\langle s, r\rangle}\left(z ; a_{-r} x, \ldots, a_{-r+t} x\right), \Pi_{t}^{\langle s, r\rangle}=\Pi_{t}^{\langle s, r\rangle}\left(z ; a_{-r}, \ldots, a_{-r+t}\right), K_{t}^{\langle r\rangle}=K_{t}^{\langle r\rangle}\left(z ; a_{-r} x, \ldots\right.$, $\left.a_{-r+t} x\right), \Pi_{t}^{\langle r\rangle}=\Pi_{t}^{\langle r\rangle}\left(z ; a_{-r}, \ldots, a_{-r+t}\right)$, a maкже $M_{t}^{\langle s, r\rangle}=M_{t}^{\langle s, r\rangle}(z), \Sigma_{t}^{\langle s, r\rangle}=\Sigma_{t}^{\langle s, r\rangle}(z)$, $M_{t}^{\langle r\rangle} u \Sigma_{t}^{\langle r\rangle}$ такие, что для любого $n \geqslant 1$ выполняются следующие равенства:

1) $R\left(x, z ; A_{n}\right)=\sum_{s=0}^{t-r} \operatorname{tr}\left(\left(K_{t}^{\langle s, r\rangle}\right)^{n} M_{t}^{\langle s, r\rangle}\right), \operatorname{per}\left(z ; A_{n}\right)=\sum_{s=0}^{t-r} \operatorname{tr}\left(\left(\Pi_{t}^{\langle s, r\rangle}\right)^{n} \Sigma_{t}^{\langle s, r\rangle}\right)$,

2) $R\left(x, z ; B_{n}\right)=\operatorname{tr}\left(\left(K_{t}^{\langle r\rangle}\right)^{n} M_{t}^{\langle r\rangle}\right), \operatorname{per}\left(z ; B_{n}\right)=\operatorname{tr}\left(\left(\Pi_{t}^{\langle r\rangle}\right)^{n} \Sigma_{t}^{\langle r\rangle}\right)$.

СлЕДСтвие 2. Производящие рядъ $\sum_{n=1}^{\infty} R\left(x, z ; A_{n}\right) y^{n}, \ldots, \sum_{n=1}^{\infty} \operatorname{per}\left(z ; B_{n}\right) y^{n}$ участвующих в теореме многочленов являются рациональными функииями от $y$.

Утверждение следствия 2 вытекает из правила суммирования геометрической прогрессии: $\sum_{n=1}^{\infty} \operatorname{tr}\left(K^{n} M\right) y^{n}=\operatorname{tr}\left((E-y K)^{-1} K M\right)$, где выражение в правой части равенства является, очевидно, рациональной функцией от $y$.

Известно (см., например, [1]), что коэффициенты разложения рациональной функции в степенной ряд удовлетворяют линейным рекуррентным соотношениям с постоянными коэффициентами. Это замечание приводит к простому эффективному вычислению многочленов теоремы для произвольно больших $n$.

Ниже мы приводим определение матриц, участвующих в формулировке теоремы, в случае $r=0$. Явное определение остальных матриц, оценки их размеров, а также доказательство теоремы 1 приведены в работе [2]. Теорема 1 является обобщением результатов работы [3], в которой аналогичные выражения приведены для ладейных полиномов и перманентов без учета цикловой структуры.

Обозначим через $N_{t}$ начальный отрезок натурального ряда, $N_{t}=\{1, \ldots, t\}$. Определим $(t+r+1)$-элементное множество $S=N_{t} \cup\left\{v_{1}, \ldots, v_{r}, w\right\}$, полученное из $N_{t}$ добавлением еще $r+1$ элементов, которые мы обозначаем формальными символами $v_{1}, \ldots, v_{r}, w$. Для всяких целых $t, r, s, d$ таких, что $t \geqslant 1,0 \leqslant r \leqslant t, 0 \leqslant s \leqslant t-r$, $0 \leqslant d \leqslant t$, обозначим через $Q_{d, t}^{\langle s, r\rangle}$ множество классов эквивалентности последовательностей $\bar{\alpha}=\left(x_{1}, \ldots, x_{s+r+d}\right), x_{j} \in S$, удовлетворяющих следующим ограничениям: 1) для всяких $i \neq j$ либо $x_{i} \neq x_{j}$, либо $x_{i}=x_{j}=w$; 2) $x_{s+r+\ell} \neq w$ для всех $\ell$, $1 \leqslant \ell \leqslant d ; 3)$ если $x_{p}=i \in N_{r}$, то $p=s+i$. При этом две последовательности $\bar{\alpha}=\left(x_{1}, \ldots, x_{s+r+d}\right)$ и $\bar{\beta}=\left(y_{1}, \ldots, y_{s+r+d}\right)$ считаются эквивалентными, если $x_{i}=y_{i}$ при $1 \leqslant i \leqslant s+r$ и $\left\{x_{s+r+1}, \ldots, x_{s+r+d}\right\}=\left\{y_{s+r+1}, \ldots, y_{s+r+d}\right\}$. Всюду далее $\{\bar{\alpha}\}$ обозначает мультимножество, составленное из элементов последовательности $\bar{\alpha}$.

Строки и столбцы матрицы $K_{t}^{\langle s, r\rangle}\left(z ; a_{-r}, \ldots, a_{-r+t}\right)=\left(k_{\bar{\alpha}, \bar{\beta}}\right)$ нумеруются элементами конечного множества $\bigcup_{d=0}^{t} Q_{d, t}^{\langle s, r\rangle}$. Для их определения зададим операцию “прибавления единицы” на множестве $S$ следующим образом: для $i \in S$ положим формально $i+1=w$, если $i$ является одним из элементов вида $t$ или $w$, положим $v_{j}+1=v_{j}$, $1 \leqslant j \leqslant r$, и прибавление единицы понимается в обычном смысле, если $1 \leqslant i \leqslant t-1$. 
Для $\bar{\alpha}=\left(i_{1}, \ldots, i_{s+d}\right) \in Q_{d, t}^{\langle s, 0\rangle}$ мы полагаем

$$
k_{\bar{\alpha}, \bar{\beta}}= \begin{cases}a_{0} z, & \text { если } \bar{\beta}=\left(i_{1}+1, \ldots, i_{s+d}+1\right)^{*}, \\ a_{i_{\ell}}, & \text { если } 1 \leqslant \ell \leqslant s, i_{\ell} \in N_{t}, \bar{\beta}=\left(i_{1}+1, \ldots, i_{\ell-1}+1,1, i_{\ell+1}+1, \ldots, i_{s+d}+1\right)^{*}, \\ a_{i_{s+\ell}}, & \text { если } 1 \leqslant \ell \leqslant d, i_{s+\ell} \in N_{t-1}, \\ 1, & \bar{\beta}=\left(i_{1}+1, \ldots, i_{s}+1,1, i_{s+1}+1, \ldots, \widehat{i_{s+\ell}+1}, \ldots, i_{s+d}+1\right)^{*}, \\ 1+a_{t}, & \text { если } t \notin\left\{i_{s+1}, \ldots, i_{s+d}\right\}, \bar{\beta}=\left(i_{1}+1, \ldots, i_{s}+1,1, i_{s+1}+1, \ldots, i_{s+d}+1\right)^{*}, \\ 0 & \text { в осли } t \in\left\{i_{s+1}, \ldots, i_{s+d}\right\}, \bar{\beta}=\left(i_{1}+1, \ldots, i_{s}+1,1, i_{s+1}+1, \ldots, i_{s+d}+1\right)^{*},\end{cases}
$$

где для всякой последовательности $\bar{\gamma}=\left(x_{1}, \ldots, x_{s+d}\right)$ мы обозначаем через $\bar{\gamma}^{*}$ ее подпоследовательность, получаемую вычеркиванием компонент $x_{i}=w$ для $i>s$. Определение матрицы $K_{t}^{\langle s, r\rangle}$ для произвольного $r \geqslant 1$ несколько более громоздко, оно приведено в [2]. Матрица $\Pi_{t}^{\langle s, 0\rangle}$ является подматрицей матрицы $K_{t}^{\langle s, 0\rangle}\left(z ; a_{0}, \ldots, a_{t}\right)$ на множестве $Q_{t}^{\langle s, 0\rangle}=\left\{\bar{\alpha} \in Q_{0, t}^{\langle s, 0\rangle} \mid w \notin\{\bar{\alpha}\}\right\}$. Матрицы $M_{t}^{\langle s, r\rangle}, \Sigma_{t}^{\langle s, r\rangle}, M_{t}^{\langle r\rangle}$ и $\Sigma_{t}^{\langle r\rangle}$ определяются из следующих равенств, являющихся уточнением равенств теоремы 1 :

$$
\begin{aligned}
R\left(x ; z ; \sum_{i=0}^{t} a_{-r+i} P_{n}^{-r+i}\right) & =\sum_{s=0}^{t-r} \sum_{\ell=0}^{r} \sum_{M_{1}} z^{\left|\left(\begin{array}{c}
\bar{\alpha}, v_{1}, \ldots, v_{r} \\
\bar{\gamma}(s+r)
\end{array}\right)\right|}\left(K_{t}^{\langle s, r\rangle}\right)^{n}\left[L\left(\bar{\alpha}, \varphi_{\bar{\beta}}\left(N_{r}\right)\right) \mid L(\bar{\gamma})\right], \\
\operatorname{per}\left(z ; \sum_{i=0}^{t} a_{-r+i} x^{i} P_{n}^{-r+i}\right) & =\sum_{s=0}^{t-r} \sum_{\ell=0}^{r} x^{(s+\ell) n} \sum_{M_{2}} z^{\left(\bar{\alpha}, v_{1}, \ldots, v_{r}\right)} \mid\left(\Pi_{t}^{\langle s, r\rangle}\right)^{n}\left[L\left(\bar{\alpha}, \varphi_{\bar{\beta}}\left(N_{r}\right)\right) \mid L(\bar{\gamma})\right], \\
R\left(x ; z ; \sum_{i=0}^{t} a_{-r+i} T_{n}^{(-r+i)}\right) & =\sum_{\bar{\gamma} \in \bigcup_{\ell=r}^{t} Q_{\ell, t}, \bar{\gamma} \supseteq N_{r}}\left(K_{t}^{\langle r\rangle}\right)^{n}\left[L\left(N_{r}\right) \mid L(\bar{\gamma})\right], \\
\operatorname{per}\left(z ; \sum_{i=0}^{t} a_{-r+i} T_{n}^{(-r+i)}\right) & =\left(\Pi_{t}^{\langle r\rangle}\right)^{n}\left[L\left(N_{r}\right) \mid L\left(N_{r}\right)\right],
\end{aligned}
$$

где $M_{1}$ и $M_{2}$ - области суммирования, определенные из условий $\bar{\alpha} \in Q_{s, t}, \bar{\beta} \in Q_{\ell, r}$,

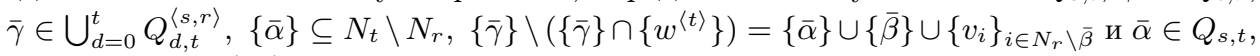
$\bar{\beta} \in Q_{\ell, r}, \bar{\gamma} \in Q_{t}^{\langle s, r\rangle},\{\bar{\alpha}\} \subseteq N_{t} \backslash N_{r},\{\bar{\gamma}\}=\{\bar{\alpha}\} \cup\{\bar{\beta}\} \cup\left\{v_{i}\right\}_{i \in N_{r} \backslash \bar{\beta}}$ соответственно. В приведенных формулах $K[L(\bar{\alpha}) \mid L(\bar{\beta})]$ - матричный элемент матрицы $K$, отвечающий последовательностям $\bar{\alpha}$ и $\bar{\beta}$. Мы полагаем $\varphi_{\bar{\beta}}(i)=i$, если $i \in \bar{\beta}$, и $\varphi_{\bar{\beta}}(i)=v_{i}$, если $i \in$ $N_{r} \backslash \bar{\beta}$. Через $\bar{\gamma}(s+r)$ обозначен начальный отрезок последовательности $\bar{\gamma}$, состоящий из первых $s+r$ ее элементов. Наконец, для последовательности $\bar{\beta}=\left(x_{1}, \ldots, x_{s+r}\right)$ такой, что $x_{i} \neq x_{j}$ при $i \neq j$, и последовательности $\bar{\gamma}=\left(y_{1}, \ldots, y_{s+r}\right)$ мы обозначаем через $\left|\left(\frac{\bar{\beta}}{\bar{\gamma}}\right)\right|$ число циклов отображения, переводящего $x_{i}$ в $y_{i}$ при $1 \leqslant i \leqslant s+r$.

Матрица $K_{t}^{\langle r\rangle}$ является подматрицей матрицы $K_{t}^{\langle 0, r\rangle}$ на множестве $\bigcup_{d=0}^{t} Q_{d, t}^{\langle r\rangle}$, $Q_{d, t}^{\langle r\rangle}=\left\{\bar{\alpha} \in Q_{d, t}^{\langle 0, r\rangle} \mid\{\bar{\alpha}\} \cap\left\{v_{1}, \ldots, v_{r}\right\}=\varnothing\right\}$. Матрица $\Pi_{t}^{\langle r\rangle}$ является подматрицей матрицы $\Pi_{t}^{\langle 0, r\rangle}\left(z ; a_{-r}, \ldots, a_{-r+t}\right)$ на множестве $Q_{t}^{\langle r\rangle}=\left\{\bar{\alpha} \in Q_{0, t}^{\langle r\rangle} \mid w \notin\{\bar{\alpha}\}\right\}$.

Автор благодарит М.Э. Казаряна за внимание к работе и полезные замечания.

\section{Список литературы}

[1] Р. Стенли, Перечислительная комбинаторика, Мир, М., 1990. [2] А. М. Каменецкий, "Общая теория цикловых ладейных полиномов и перманентов прямоугольных матриц с приложениями к циркулянтам и тёплицевым матрицам", Материаль V Междунар. сем. по дискрет. матем. и ее прилож. (31 янв. - 2 февр. 1995 г.). [3] А. М. Каменецкий, УМН, 60:3 (2005), 177-178.

А. М. Каменецкий (А. М. Kamenetskii)

E-mail: kamenetsky_a@list.ru
Представлено С. К. Ландо Принято редколлегией 07.10 .2007 